\title{
The effect of a moving ground on the flow and aerodynamic noise behaviour of a simplified high-speed train bogie
}

\author{
J. Y. Zhu $u^{a, c *}$, Z. W. Hua , D. J. Thompson ${ }^{b}$ \\ a Aerodynamics and Flight Mechanics Research Group, Faculty of Engineering and the Environment, \\ University of Southampton, Southampton SO17 1BJ, United Kingdom \\ b Institute of Sound and Vibration Research, Faculty of Engineering and the Environment, University of \\ Southampton, Southampton SO17 1BJ, United Kingdom \\ c Institute of Railway and Urban Mass Transit, Tongji University, Shanghai 200092, People's Republic of \\ China
}

\begin{abstract}
Aeroacoustic sources around the train bogie area are particularly important but involve complex flow. In this study, a numerical investigation is presented of the effect of a moving ground on the flow and aeroacoustic noise behaviour of a train bogie. These make use of the delayed detached-eddy simulation combined with an acoustic analogy for noise prediction. First the flow around an isolated wheelset (1:5 scale) is calculated when it is in proximity to the ground and this is compared with wind-tunnel measurements to verify the simulation. Then the flow and aerodynamic noise behaviour of a simplified high-speed train bogie at scale 1:10 with and without the ground underneath are studied numerically. It is found that a highly turbulent flow is generated within the bogie cavity and the ground increases the noise levels by $6-8 \mathrm{~dB}$ due to a combination of acoustic reflection from the ground and modifications to the flow.
\end{abstract}

Keywords: railway noise; flow behaviour; aerodynamic noise; simplified train bogie; moving ground; sound radiation

\section{Introduction}

For high-speed trains, it is generally accepted that the aerodynamic noise becomes an important source at running speeds over about $300 \mathrm{~km} / \mathrm{h}$ [1-3]. The main aeroacoustic sources on high-speed trains identified by various studies [2,4] are the bogie, the pantograph, the recess of the pantograph, the inter-coach spacing, etc. As one of the main aerodynamic noise sources of high-speed trains, the bogie is a complex structure

*Corresponding author. Email: j.zhu@soton.ac.uk; zhujianyue@tongji.edu.cn 
containing many components with surface shape variations and discontinuities, thus the flow generated around it is highly turbulent [2,3] and some methods, like mounting the bogie skirts on the bogies, were studied to modify the flow developed there [5]. Moreover, the bogie region is situated close to the ground and is moving at high speed relative to the ground so it is necessary to consider the influence of the ground on the aerodynamic and aeroacoustic behaviour around the bogie areas.

Most aeroacoustic studies on the bogie area have involved wind-tunnel and trackside experiments. A 1:7 scale simplified bogie located in the bogie cavity of a train model was used by Yamazaki et al. [6] to study the aerodynamic noise generated from a bogie by using a directional microphone array in a low-noise wind tunnel. The flow velocity profile under the train was adjusted and the noise predictions were validated by field-test results. To locate the main sources of noise in the bogie area and to characterize the unsteady sources, an experiment was carried out using a 1:25 scale model of an ICE3 (inter city express) by Lauterbach et al. [7] in an aeroacoustic wind tunnel. A microphone array was positioned outside the flow in the open-jet wind tunnel. The interest was focused on the bogie cavities and the gaps between the vehicles. Based on these measurements, noise maps from the bogie area were obtained and modifications for noise reduction were proposed.

In running tests it is more difficult to separate rolling noise and aerodynamic noise from the bogie region by experimental methods. Using a star-shaped microphone array, the noise source maps from a TGV (train à grande vitesse) Duplex at $350 \mathrm{~km} / \mathrm{h}$ were presented by Poisson et al. [8]. They showed that the significant aerodynamic noise was generated in the leading bogie region which can be attributed to a large number of flow separations and interactions with high-level turbulence developed there.

As considerable computational resources are required for numerical calculations, aerodynamic noise prediction is still infeasible in an industrial context using flow simulations [9]. The authors have therefore focused on a simplified bogie. In [10] the aerodynamic and aeroacoustic behaviour of the flow past an isolated wheelset was studied and in [11] this was extended to the flow and flow-induced noise behaviour of an isolated simplified bogie. The results showed that vortex shedding and flow separation around the geometries were the key factors for the aerodynamic noise generation. It is 
evident that a good understanding of the sound sources and their physical characteristics is essential to find good solutions to control the aerodynamic noise. This paper aims to extend this study to investigate numerically the effects of a moving ground on the aerodynamic and aeroacoustic behaviour of the flow passing the same reduced scale simplified high-speed train bogie which is now located inside the bogie cavity under the train body. First, an isolated wheelset case in proximity to a stationary ground is used for verification of the numerical procedure and to see the influence of the ground on the flow development.

\section{Numerical method}

Numerical simulations are carried out using a two-stage strategy of computational fluid dynamics (CFD) and computational aeroacoustics (CAA) methods. Aerodynamically, high-speed trains are operating within the low Mach number flow regime, up to about 0.20.3. Thus, the compressibility effects may be neglected in studying the hydrodynamics of the flow field. Moreover, at low Mach number the dominant noise sources are the dipole sources generated from wall pressure fluctuations, which can be predicted essentially through incompressible flow modelling [12]. For an open cavity case at a Mach number of 0.15 as an example, the sound source intensities on the cavity wall were in good agreement between the incompressible and compressible flow solutions which were applied as the noise sources in the acoustic analogy method [13]. In the present study, the incoming flow simulated here is at a low Mach number of 0.09 (corresponding to 30 $\mathrm{m} / \mathrm{s}$ ) and thereby the unsteady, incompressible Navier-Stokes equations are used to solve the flow field. The open source software OpenFOAM-2.2.1 is employed for flow simulations. A scheme of second-order accuracy is used for the convective and diffusive terms of the spatial derivatives and the temporal discretization follows a second-order fully implicit scheme. The delayed detached-eddy simulation (DDES) based on the Spalart-Allmaras turbulence model is applied for the flow calculations [14]. As a hybrid technique, detached-eddy simulation combines unsteady Reynolds-averaged NavierStokes (URANS) modelling in the near-wall regions to resolve the boundary layer, with large-eddy simulation (LES) in the massive separated outer flow regions to capture the large-scale structures. DDES has been developed to avoid grid-induced separation caused by modelled stress depletion and to preserve the Reynolds-averaged Navier- 
Stokes (RANS) mode throughout the boundary layer. Different with the classical Ffowcs Williams-Hawkings (FW-H) acoustic analogy method used in [10,11], the near-field unsteady flow calculations provide acoustic sources on a permeable surface for far-field noise predictions based on the convective FW-H formulation introduced in [15].

Note that in an incompressible flow solver, as used here, the sound speed is effectively infinite. However, the error introduced is relatively small for the current cases at a low Mach number. For example, Orselli et al. [16] investigated the case of flow passing over a circular cylinder at a Mach number of 0.2 based on the acoustic analogy method. Using the incompressible flow field solutions as the noise source data, they found that the noise predicted using an off-body permeable surface was slightly higher than that calculated on the cylinder wall surfaces.

\section{Numerical verification by an isolated wheelset case}

A particle image velocimetry (PIV) experiment for an isolated wheelset case has been performed in a wind tunnel. A 1:5 scale wheelset model with a cylindrical axle and simple discs for the wheels made of aluminium was attached on the mounting struts fixed on the wind-tunnel roof and a gap of $40 \mathrm{~mm}$ between the wheelset and the ground was considered to represent approximately the distance of the wheels above the ground when running on the rails. The ground is stationary and the rails are neglected here. The PIV measurements are compared with numerical simulations for this case to verify the calculated effects.

\subsection{Experimental measurements}

Figure 1 shows the front view of the experimental setup of the isolated wheelset case in the $7^{\prime} \times 5^{\prime}(2.1 \mathrm{~m} \times 1.5 \mathrm{~m})$ wind tunnel at the University of Southampton with the PIV system installed. This system consists of a Gemini PIV 15 laser with two Tempest Ng:YAG lasers of $125 \mathrm{~mJ} /$ pulse at a wavelength of $532 \mathrm{~nm}$, and a TSI PowerView CCD camera (2048 2048 pixels resolution) to capture the images. The mid-span plane of one wheel along the streamwise direction was measured using PIV. Figure 2 displays the streamwise mean velocity field in the wake region of the wheel. Asymmetric flow 
conditions are apparent around the wheel wake region in Figure 2(b) since an angle of $9.7^{\circ}$ between the central mean streamline and the wheel equatorial axis is observed downward towards the ground. Such an influence of the ground is also confirmed by the CFD simulation presented below.

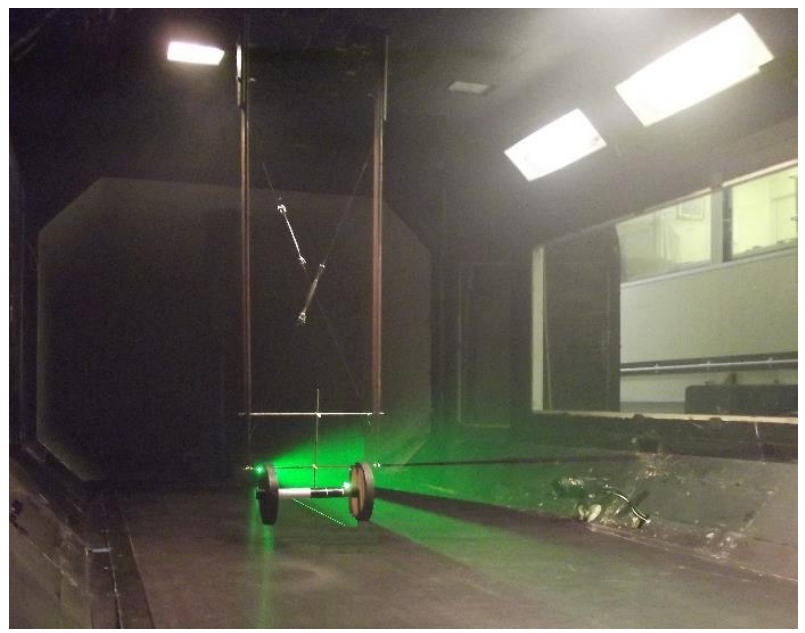

Figure 1. Front view of the PIV experimental setup of isolated wheelset case

(a)

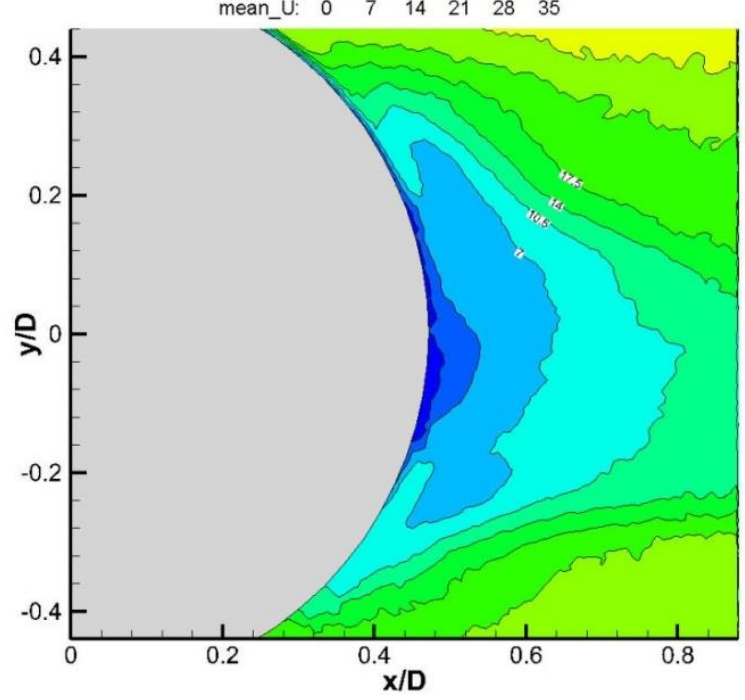

(b)

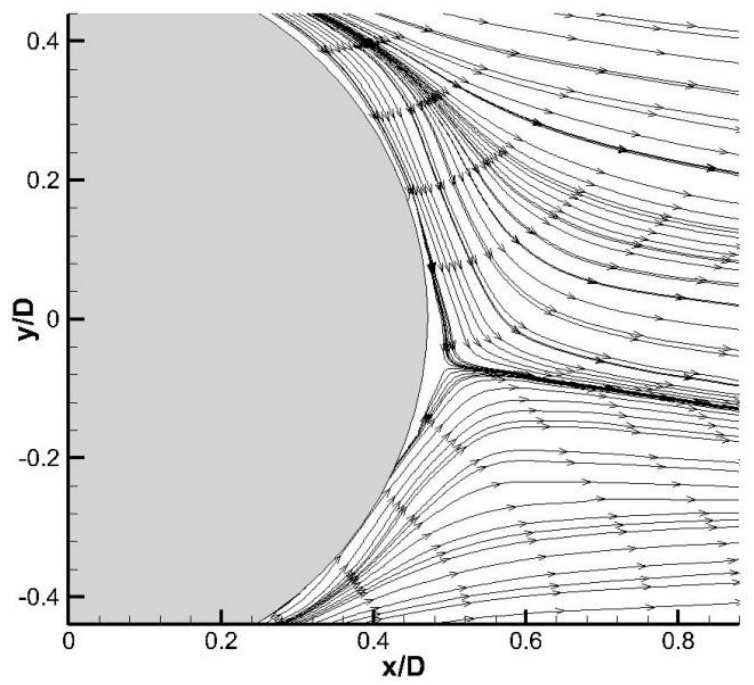

Figure 2. Streamwise mean velocity field from PIV experiments (free stream flow speed of $30 \mathrm{~m} / \mathrm{s}$ and wheel diameter of $184 \mathrm{~mm}$ ): (a) mean velocity; (b) mean streamlines.

\subsection{Numerical simulation}

Computations are carried out based on the dimensions and experimental conditions from the 7' $\times 5$ ' wind tunnel. Corresponding to the dimensions of the wind tunnel working section 
( $2.1 \mathrm{~m}$ wide $\times 1.5 \mathrm{~m}$ high $\times 4.4 \mathrm{~m}$ long), the computational domain is built and illustrated in Figure 3. Here $D(=184 \mathrm{~mm})$ is the diameter of the $1: 5$ scale wheel and $h(=40 \mathrm{~mm})$ is the gap between the bottom of the wheel and the ground. The corners in the tunnel are simplified to be rectangular. In the simulation, the wheelset and the ground are both defined as stationary no-slip walls. The symmetry plane at the axle mid-span is set to a symmetry boundary condition which assumes no flux of any quantity across it. Equivalent to a zero-shear slip wall, the tunnel ceiling and the side wall of the domain are also defined as symmetry boundary conditions to avoid the need for a high grid density required to resolve the wind-tunnel boundary layer. The upstream boundary is set to a velocity inlet with a normal velocity of $30 \mathrm{~m} / \mathrm{s}$. As the wind tunnel is of low turbulence intensity $(\approx 0.3 \%)$, a low turbulent viscosity ratio of unity is applied for the inlet flow. The downstream boundary is defined as a pressure outlet with a gauge pressure of zero. The physical timestep size is $5 \times 10^{-6} \mathrm{~s}$ initially and then increases to $1 \times 10^{-5} \mathrm{~s}$ which gives the temporal resolution for the simulation with respect to the Courant-Friedrichs-Lewy (CFL) number of less than 2 within the computational domain. The Reynolds number (based on the freestream properties and the axle diameter) in the 1:5 scale wheelset case is 72,000 which is within the subcritical Reynolds number regime of cylinders [17]. As is well known, for a uniform stream passing over the axle of cylindrical shape, the boundary layer separation is laminar and vortex shedding occurs in the wake area with a Strouhal number of about 0.2 in the subcritical flow regime. A fully structured mesh is generated in the entire domain and the total number of grid points is 19.4 million.

(a)

(b)
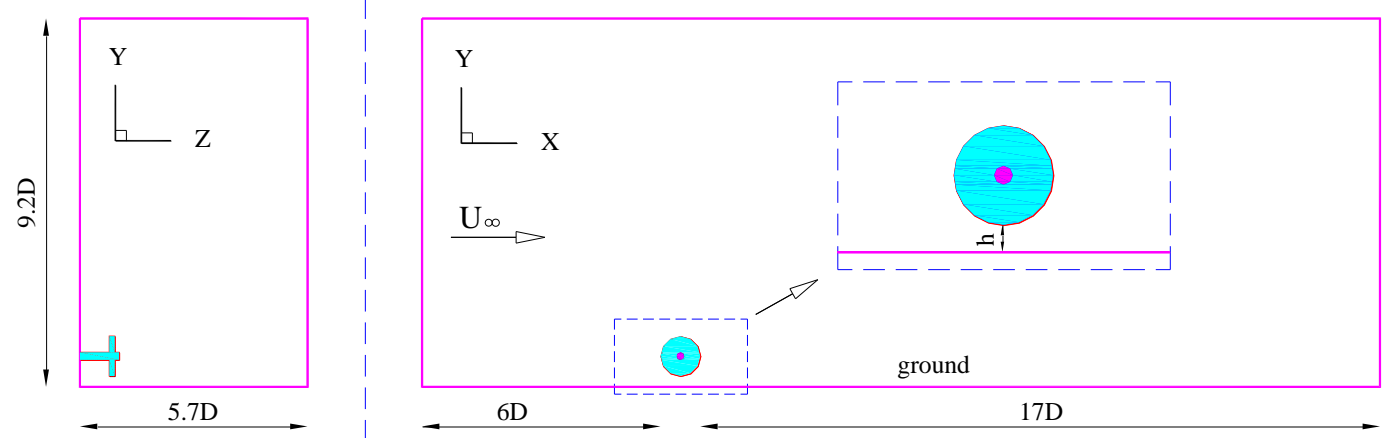

Figure 3. Sketch of the computational domain with the wheelset (not to scale): (a) front view; (b) side view. 
The data from the CFD analysis corresponding to the PIV experiment are displayed in Figure 4. The computed flow patterns shown in this figure are qualitatively similar to those from the PIV measurements (Figure 2). Likewise, asymmetric flow conditions is observed in Figure 4(b) with an average angle of $9.9^{\circ}$ between the central mean streamline and the wheel equatorial axis downward to the ground. This is believed to be the result of the proximity of the ground: when the ground is fixed, a boundary layer develops on the ground surface and interferes with the separated shear layer from the bottom side of the wheelset. By comparison, a symmetrical flow pattern along the wheelset horizontal central plane is developed for the isolated wheelset case without the ground [18].

(a)

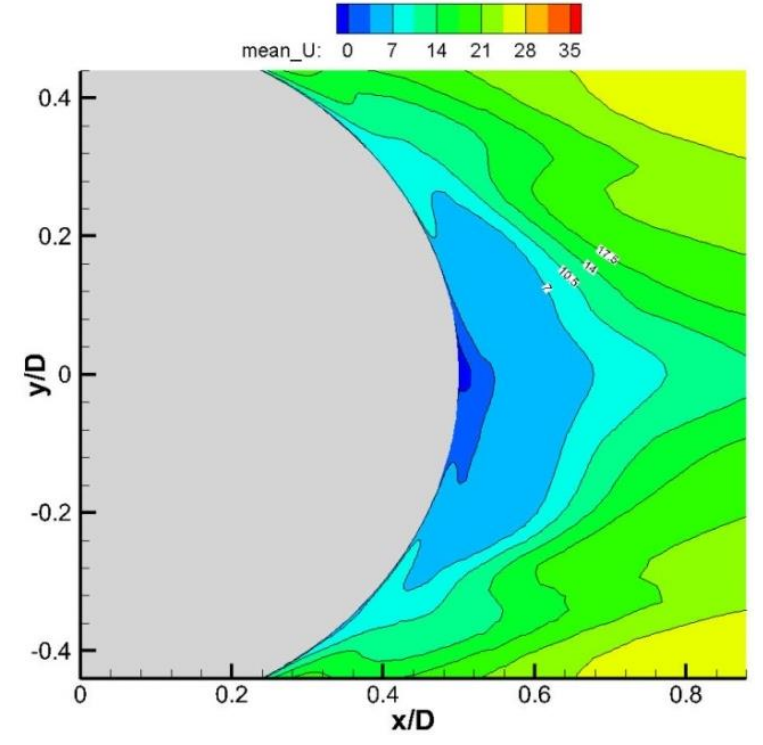

(b)

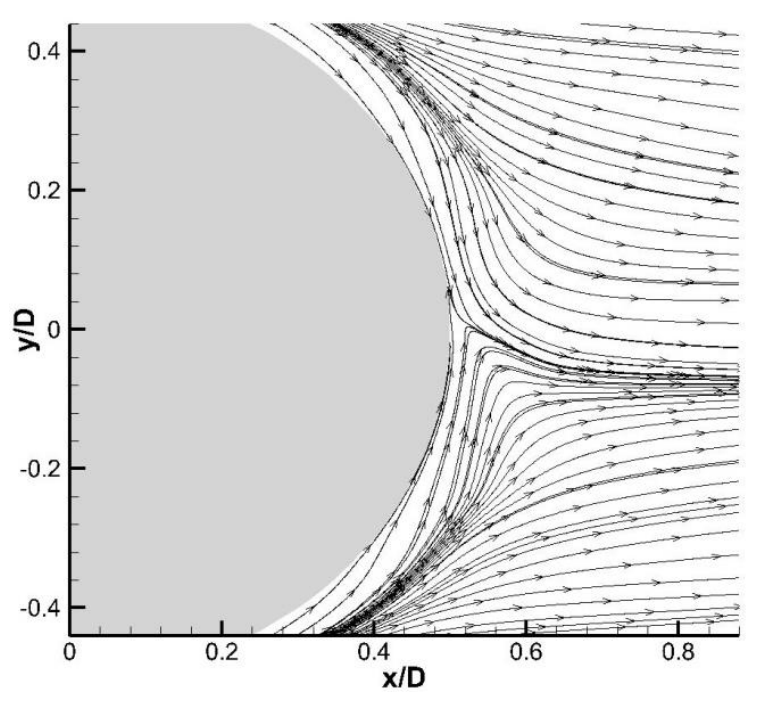

Figure 4. Streamwise mean velocity field from CFD simulations: (a) mean velocity; (b) mean streamlines.

Figure 5 compares the mean velocity profiles (normalised by the free stream velocity) behind the wheel of the isolated wheelset at $x / D=0.6$ in the wheel mid-span between the calculation and experiment results. It can be seen that the CFD simulation successfully captured the streamwise and vertical mean wake profiles of the wheelset. Therefore, the above results give a reasonable indication that the wheel wake pattern is properly identified by the numerical simulation by its good agreement with the experimental measurements. 
(a)

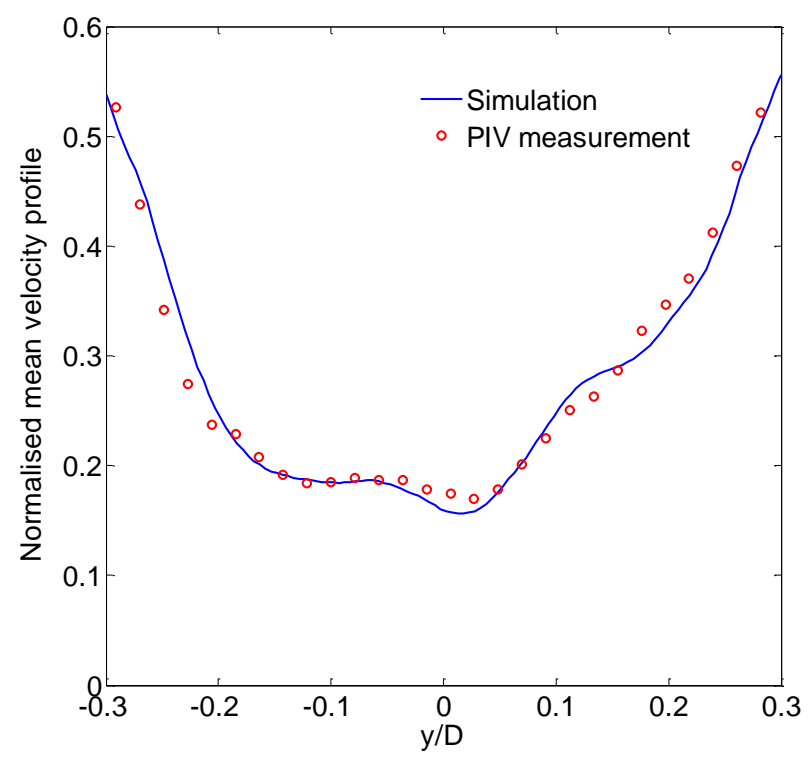

(b)

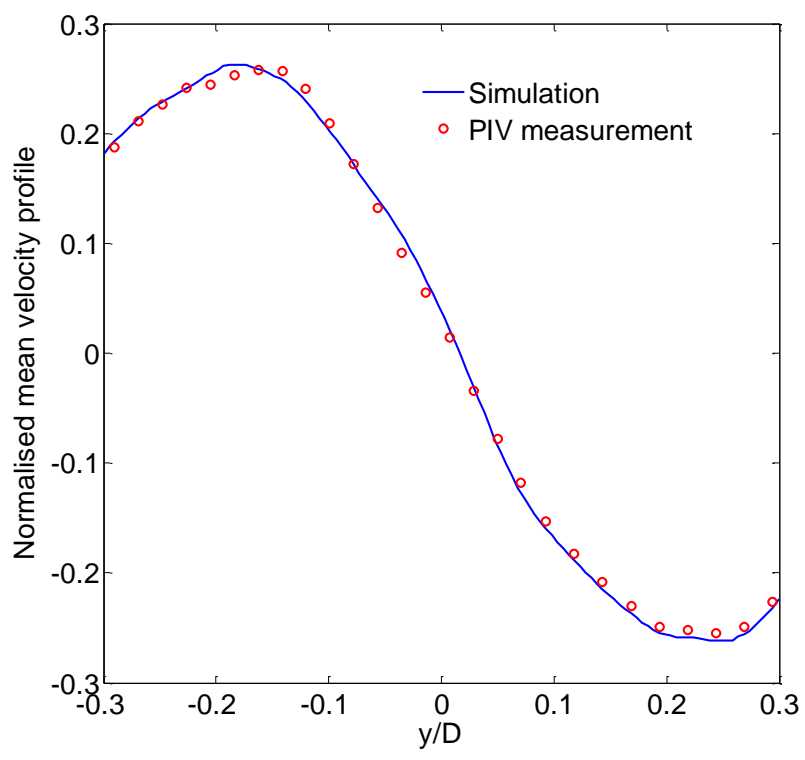

Figure 5. Comparisons of streamwise and vertical mean velocity profiles at $x / D=0.6$ : (a) streamwise; (b) vertical.

\section{Simulation setup}

In order to study the influence of the ground on the flow and flow-induced noise behaviour around the bogie region, two cases, with and without the ground, are simulated to see the difference between them. These both consist of a simplified bogie at 1:10 scale (as in [11]) which is now located within a cavity on the underside of a train car body. The flow within the bogie cavity is characterized by vortex shedding, turbulent wake/structure and wake/wake interactions. To reduce the simulation cost, the most representative components that generate vortex shedding and turbulent wake, such as the wheels, axles and the frame, are kept in the simplified bogie model and the other details are removed. Thus, the high frequency sources may be lost, but the main lower frequency behavior is expected to be retained. Figure 6 shows the model of the simplified bogie geometry inside the bogie cavity with the ground underneath. The computational domain has dimensions of $20.7 D, 5.8 D$ and $6.3 D$ (where $D$ is the $1: 10$ scale wheel diameter) along the streamwise $(x)$, vertical $(y)$ and spanwise $(z)$ directions, respectively. The simplified bogie is the same bogie as in [11]: the axle has a diameter $(d)$ of $17.5 \mathrm{~mm}$ and the wheel has a diameter $(D)$ of $92 \mathrm{~mm}$. The wheelbase (centre-to-centre length of two axles) is $252 \mathrm{~mm}$ which is about 14 times the axle diameter. The gap between the bottom of the wheel and 
the ground floor is $25 \mathrm{~mm}$ to represent the distance of the wheels above the ground when running on the rails, although the rails are omitted from the model. The carbody underfloor surfaces are $4 \mathrm{~mm}$ above the bogie horizontal central plane and the carbody sides are $130 \mathrm{~mm}$ away from the bogie centre. The geometry is symmetrical about the axle mid-span where the influence of the three-dimensional flow from the wheel and frame is small; therefore it is reasonable to include only half of the geometry and make use of the symmetry to reduce the computational cost. For the case without the ground, the computational domain is similar to the case with the ground except the dimension in the vertical direction is $11 D$ (where $D$ is the wheel diameter) and the bogie centre is located centrally in the domain in the vertical direction.

(a)

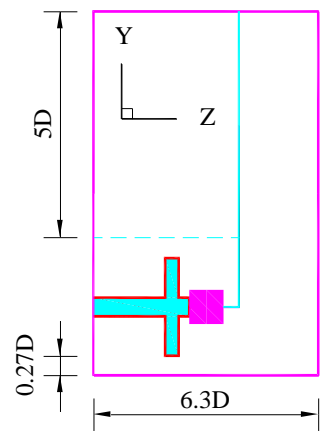

(b)

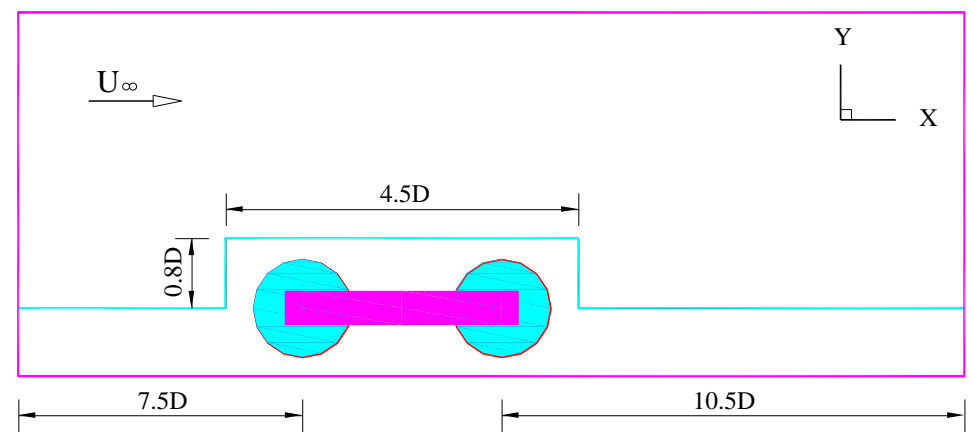

Figure 6. Sketch of the computational domain of bogie inside the cavity with ground underneath (not to scale): (a) front view; (b) side view.

Because of the large calculations required for the unsteady flow, a rigorous grid convergence study for a complex geometry case is difficult to achieve. Based on the mesh refinement study for the cylinder case in [11,18] and the mesh topology of the 1:5 scale isolated wheelset case discussed previously, a fully block-structured mesh is generated around all geometries. The mesh around the bogie is displayed in Figure 7. The distance from the bogie, cavity wall and ground surface to the nearest grid point is set as $1 \times 10^{-5} \mathrm{~m}$ and stretched with a growth ratio of 1.1 inside the boundary layer. This yields a maximum value of $y^{+}$(the dimensionless first-cell spacing, $y^{+}=\frac{y u_{\tau}}{v}$ where $y$ is the distance from the wall, $u_{\tau}$ the friction velocity and $v$ kinetic viscosity) less than 1 for all cases. This ensures that the boundary layer is resolved properly and the turbulence model employed can account for the low-Reynolds number effects inside the viscous sublayer. This grid generation strategy results in the structured meshes in the entire 
domain with a total number of grid points of 36.5 million for the case without the ground and 39.5 million for the case with the ground underneath. The same mesh topology was employed previously for the isolated wheelset and bogie cases in which good agreements were achieved between numerical simulations and experimental measurements for the radiated far-field noise $[10,11,18]$.

(a)

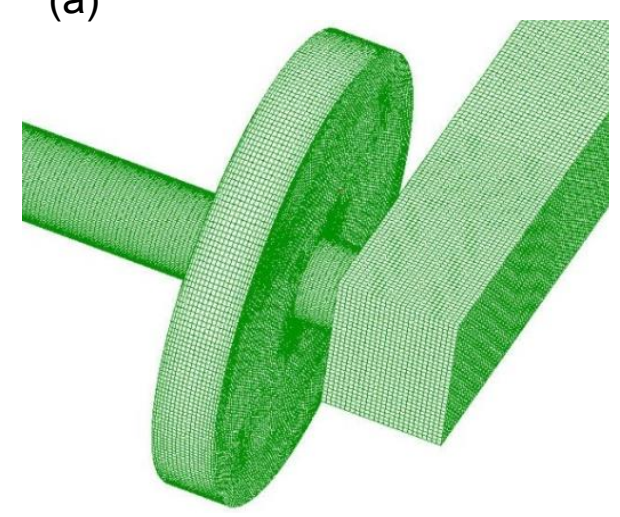

(b)

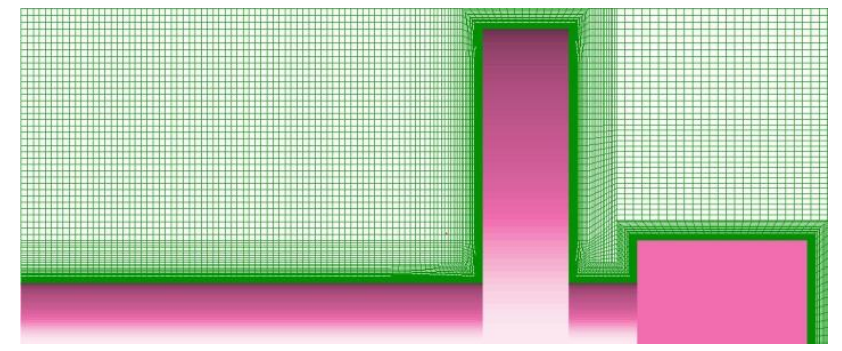

Figure 7. Structured mesh topology around the bogie: (a) bogie surfaces; (b) wheelset mid-plane.

The boundary conditions applied are as follows: the upstream inlet flow is represented as a steady uniform flow of $30 \mathrm{~m} / \mathrm{s}$ with a low turbulence intensity. Thus, in the Spalart-Allmaras turbulence model used here, the production term of the transport equation is activated and the eddy viscosity comes into equilibrium with the velocity field rapidly as soon as the fluid enters the boundary layer. Additionally, the top, axle mid-plane and side boundaries are specified as having symmetry boundary conditions which are equivalent to zero-shear slip walls; a pressure outlet with zero gauge pressure is imposed at the downstream exit boundary. For the case with the ground underneath, the wheelsets are assumed to be rotating and all wheelset surfaces are defined as moving no-slip walls with the corresponding rotation velocity imposed on the solid surfaces. The ground is set as a no-slip wall moving with the inflow velocity and the other solid surfaces are defined as stationary no-slip walls. For the case without the ground, all solid surfaces are defined as stationary no-slip walls and the wheelset is assumed not to be rotating.

Simulations are run with a physical timestep size of $5 \times 10^{-6} \mathrm{~s}$ initially increasing to $1 \times 10^{-5} \mathrm{~s}$ which gives an adequate temporal resolution for the implicit time marching 
scheme used with a CFL number of less than 1 within most of the computational domain and a maximum value of 2. The Reynolds number (based on the freestream properties and the axle diameter) of the bogie-inside-cavity cases is 36,000 , which is within the subcritical flow regime of cylinders [17]. Compared with the isolated wheelset case investigated earlier, the vortex shedding effect will be weaker for the wheelset located in the bogie cavity. Therefore, the results from the bogie-inside-cavity cases are expected to be applicable to full scale.

\section{Influence of ground on flow development}

In order to investigate the flow-field characteristics, the simulation results of the instantaneous iso-surfaces of $Q$-criterion and the vorticity fields are displayed to get an overview of the unsteady flow developed around the geometries.

Figure 8 visualises the vortical structures of the turbulent flow represented by the isosurfaces of the second invariant of the velocity gradient, $Q$, plotted at a normalised value of 50 (based on $Q /\left[\left(U_{\infty} / D\right)^{2}\right]$, where $U_{\infty}$ is the freestream velocity and $D$ is the wheel diameter) for the case with the ground underneath. Figure 8(a) shows that the vortices shed from the upstream geometries impinge on the downstream ones, generating a highly turbulent wake behind the bogie. Subsequently, all vortices impinge on the cavity rear wall, deform largely and are merged into the eddies formed downstream behind the cavity. It can be observed from Figure 8(b) that different scales of vortices are generated between the upstream wheelset and cavity top wall as the various flow interactions and flow impingements occur there. Some vortices are convected downwards and impinge on the ground. Moreover, compared with the flow developed around the bogie, a higher level of flow-field unsteadiness is generated in the wake close to the cavity rear wall; nevertheless, it dissipates rapidly downstream as the flow development is impeded by the wall surface of the vehicle carbody. 
(a)
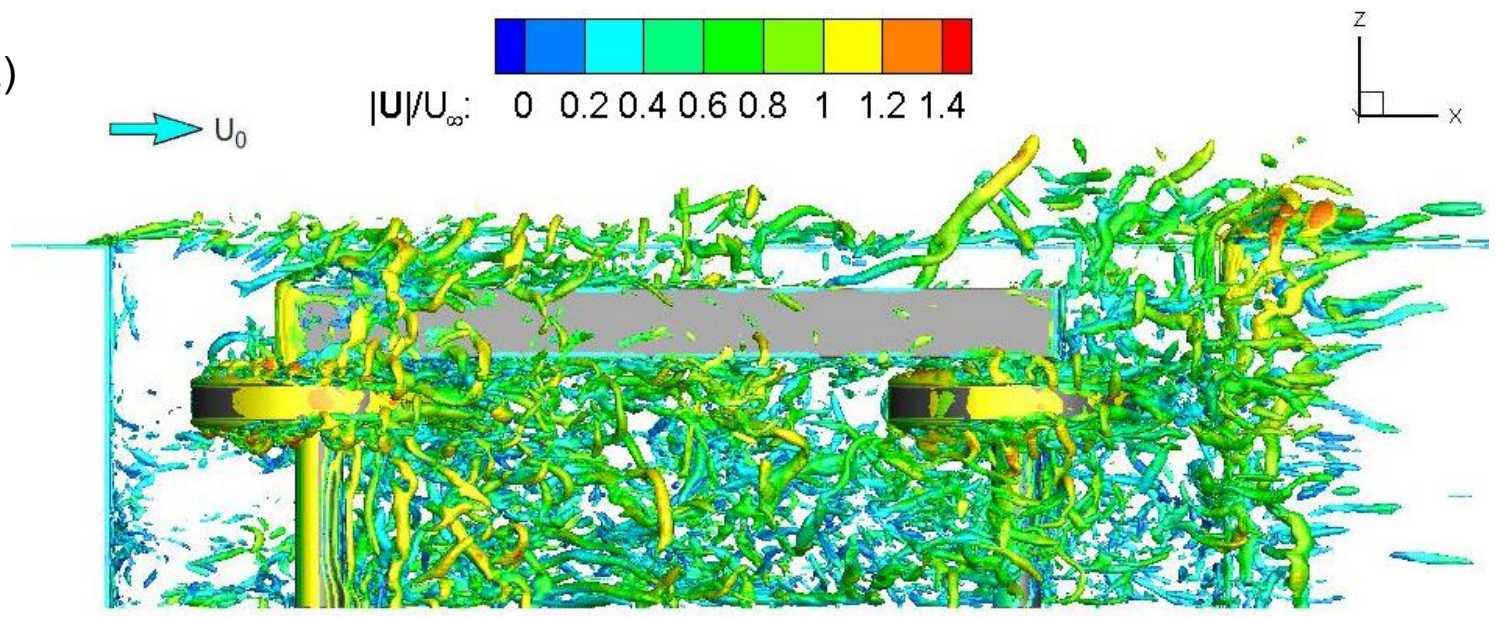

(b)

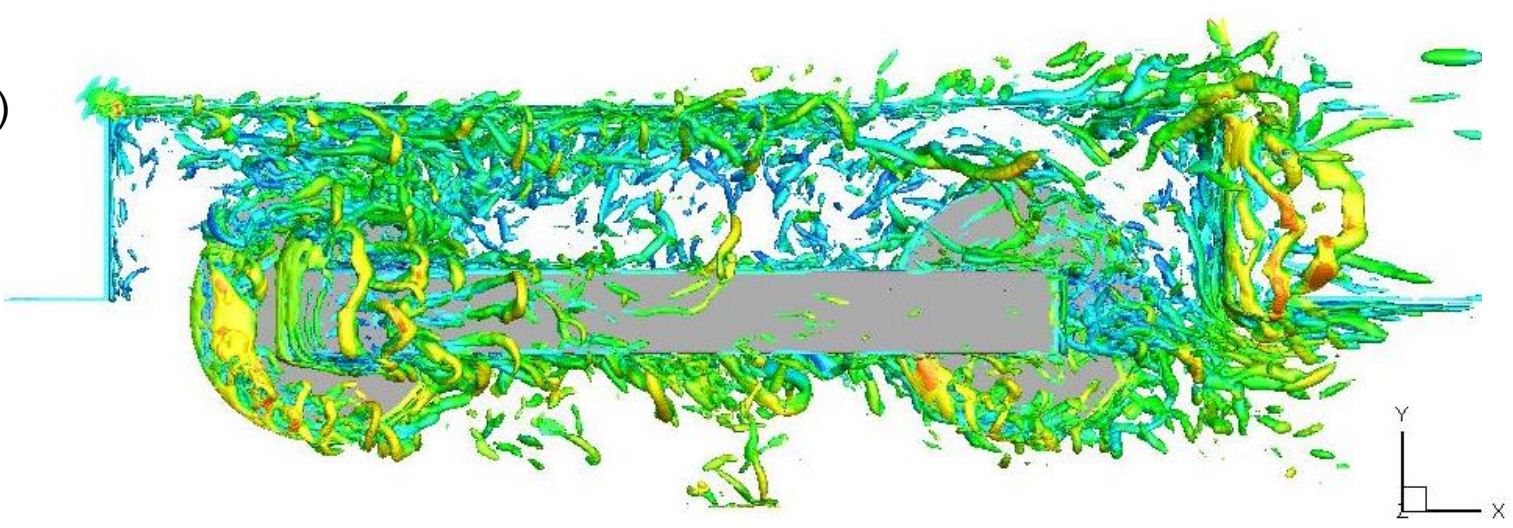

Figure 8. Iso-surfaces of the instantaneous normalised $Q$-criterion (case with the ground): (a) bottom view; (b) side view. 
(a)

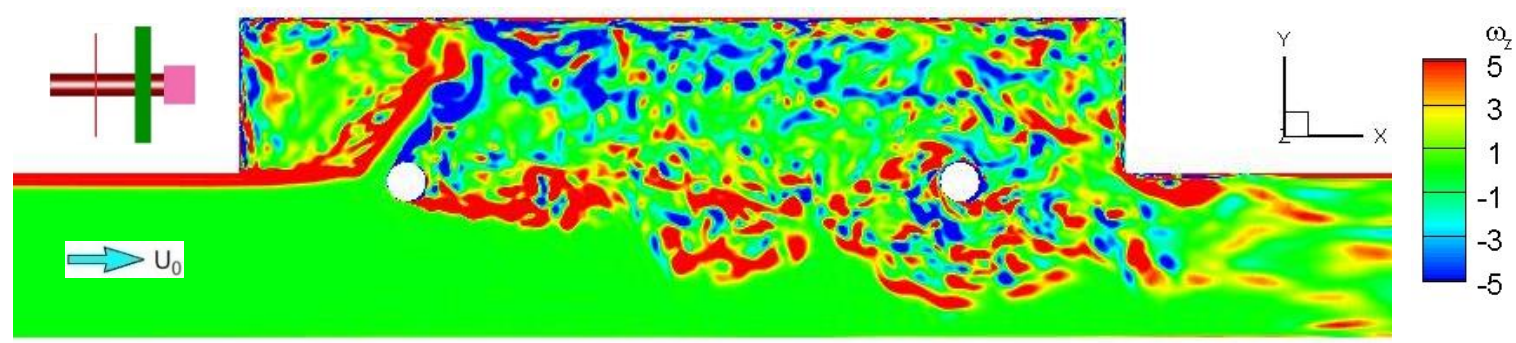

(b)

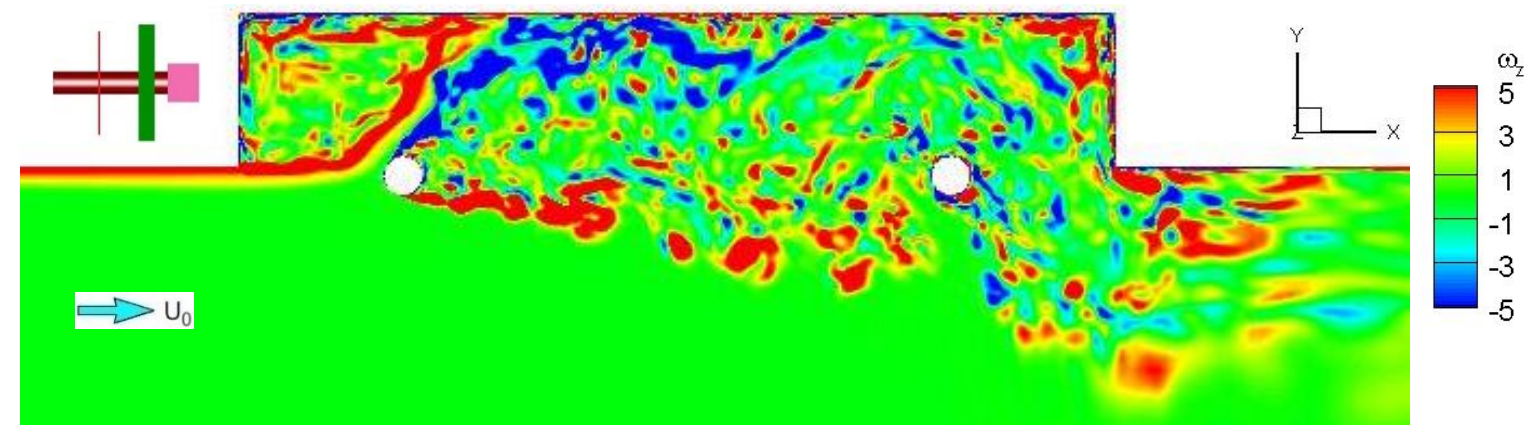

Figure 9. Contours of the instantaneous spanwise vorticity field in the half axle midplane (side views): (a) with the ground; (b) without the ground.

Figure 9 depicts the instantaneous non-dimensional spanwise vorticity field $\left(\omega_{z}=\right.$ $(\partial V / \partial x-\partial U / \partial y) D / U_{\infty}$, where $D$ is the wheel diameter) in the plane midway between the wheel inner surface and the axle centre for the cases with and without the ground. This shows that for both cases a shear layer is shed from the cavity leading edge and bent upwards rapidly in the streamwise direction. As a consequence, the boundary layer on the axle surface cannot be fully developed, and thus no vortex is formed in the region behind the separation point and the subsequent organized vortex shedding seen for the isolated wheelset case [10] is lost. Then, all vortices are mixed up and impinge on the cavity top walls and rear corners, resulting in a highly irregular and unsteady flow formed there. Compared with the flow field of the case without the ground, more eddies with various scales are developed inside the bogie region for the case with the ground underneath (especially around the rear axle) since the moving ground and the rotating wheelsets generate more flow interactions.

\section{Influence of ground on sound radiation}

The results from the CFD calculations are used to predict the far-field noise in a Ffowcs Williams-Hawkings solver. The CFD simulations were run for $1.12 \mathrm{~s}$ corresponding to 17 
times the flow-through time. The length of the time signal used as input for noise calculation corresponds to the last $0.52 \mathrm{~s}$ of the computations, by which time the transient flow field has become statistically steady. The power spectral density (PSD) is computed from the predicted far-field noise time history by Welch's method and averaged over $50 \%$ overlapping segments using a Hanning window applied to 5 segments, giving a frequency resolution of $6 \mathrm{~Hz}$. The overall sound pressure level (OASPL) is calculated from the PSD in a frequency range between $120 \mathrm{~Hz}$ and $2 \mathrm{kHz}$.

In the classical FW-H method, the wind-tunnel problem should be transformed into a moving-observer problem where the observer is assumed to be moving with the source at a constant speed in an environment at rest $[19,20]$. In order to improve the calculation efficiency, the observer needs to remain stationary in the wind-tunnel case and the moving mean flow should be taken into account in the computation. Thus, based on the convective $\mathrm{FW}-\mathrm{H}$ formulation [15], a noise prediction code has been implemented to calculate the far-field noise [18]. The time-dependent flow field and the geometry information on panels (surface elements) are obtained from the CFD simulation data.

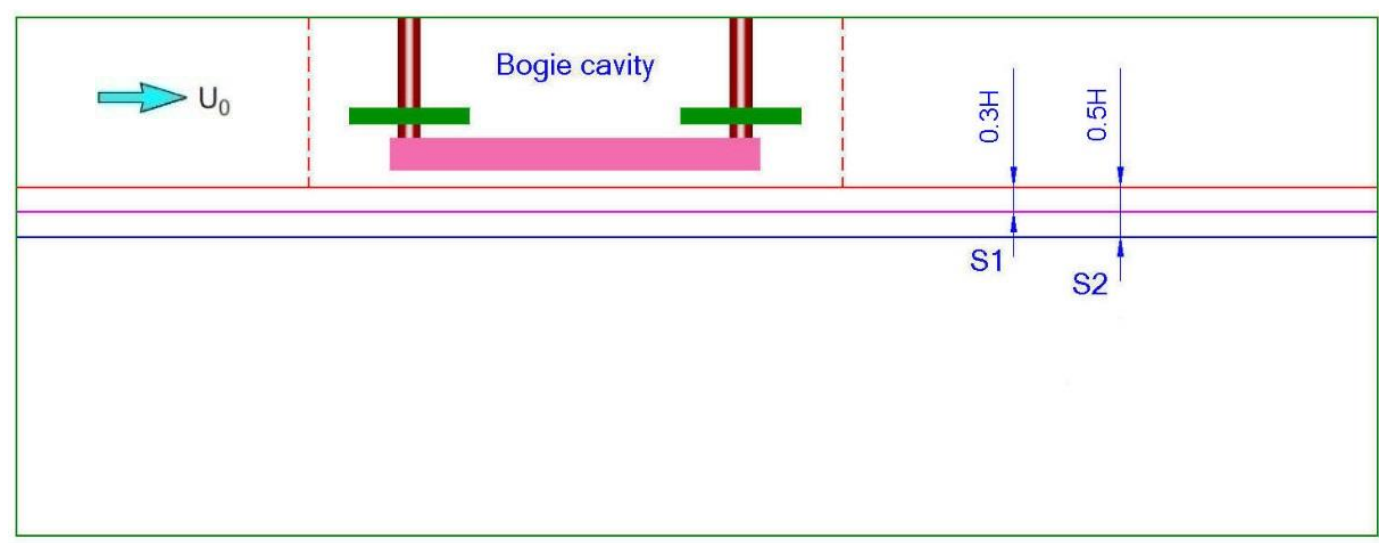

Figure 10. Sketch of porous surfaces used for FW-H integration (top view, not to scale)

Due to the complexity of the bogie geometry, a conventional FW-H method based on the solid surfaces has the disadvantage that it does not correctly represent the sound radiation from hidden surfaces. The radiated noise is therefore calculated using the acoustic analogy implemented on a permeable surface located at a distance parallel to the carbody sidewall, extending over the whole height and length of the domain. Two porous surfaces (S1 and S2) are used for the FW-H integration as illustrated in Figure 10. The gap between the porous surface (S1) and the carbody side wall is $0.3 H=21 \mathrm{~mm}$ 
(where $H$ is the bogie cavity depth of $70 \mathrm{~mm}$ ) and that for the porous surface (S2) is $0.5 H=35 \mathrm{~mm}$. These two gap distances are chosen based on noise predictions in [21] on a rectangular cavity flow, where the cases with different distances to the cavity top wall were simulated and the numerical results from the FW-H method were in good agreement with the direct numerical simulations. The mesh between the two permeable surfaces is kept fine to ensure that high resolution flow calculations are performed around these surfaces. Consequently, a compromise is considered between the requirement to keep the permeable surface as far as possible from the turbulence around the geometries and the need to avoid increasing the mesh grid points too much. In the current calculations, there are 129,666 panels on the permeable data surface for the case without the ground and 140,988 panels for the case with the ground underneath. The image method introduced in [22] is used to account for the sound reflection of the ground. Assuming no sound absorption is introduced by the ground, an equivalent noise source is located symmetrically with respect to the ground surface and, together with the original sources, this generates a sound field corresponding to the symmetry plane.

Figure 11 compares of the far-field noise spectra of the case without the ground based on the numerical simulations on the symmetrical half bogie (shown in Figure 10) using the permeable and solid data surfaces in the $\mathrm{FW}-\mathrm{H}$ method. The receiver is located $2.5 \mathrm{~m}$ away and $0.35 \mathrm{~m}$ above the bogie centre. This shows that the noise predictions of the FW-H integrations on the two permeable surfaces (S1 and S2) are very similar, indicating that the influence of the placement of these two surfaces on calculating the farfield acoustic signals is negligible. However, compared with the noise generated from all wall surfaces, the amplitude of noise predicted by the permeable integration surfaces is around $20 \mathrm{~dB}$ higher below $120 \mathrm{~Hz}$. This can be attributed to unphysical results generated in the noise predictions using the flow data obtained on non-closed permeable surfaces. If the surface is not closed, the integration in the noise calculation is unbalanced, especially at low frequency. It is not possible to close the permeable surfaces in the current case due to the turbulent boundary layer developed on the train wall, although they have been extended up- and down-stream as far as possible in an attempt to minimise the effect. Similar influences of the open control surface were also observed in the noise predictions of a simplified landing gear using FW-H method by Spalart et al. [9]. Therefore, the frequency range between $120 \mathrm{~Hz}$ and $2 \mathrm{kHz}$ will be used for noise prediction, and within this frequency range, the noise generated from the permeable 
integration surface (S2) is $2.6 \mathrm{~dB}$ higher than that from all solid surfaces whereas that from the permeable surface $\mathrm{S} 1$ is $3.1 \mathrm{~dB}$ higher. These differences can be attributed to the pseudo-sound generated by the turbulent eddies passing through the permeable surface and the result at high frequencies for $S 2$ is closer to that for the rigid surfaces than is the case for S1. Thus, keeping the permeable surface further away from the geometries can reduce the pseudo-sound produced from the hydrodynamic perturbations; however, it will increase the computation cost greatly as the grids between the train and the permeable surface should be kept fine enough to get physically correct results. The quadrupole noise generated by the turbulent flow around the geometries is also captured by the surface integrals on the permeable data surfaces but its contribution is expected to be rather small for current cases at low Mach numbers. To reduce the computation cost, the permeable surface (S1) is used for the noise prediction of the case with the ground underneath.

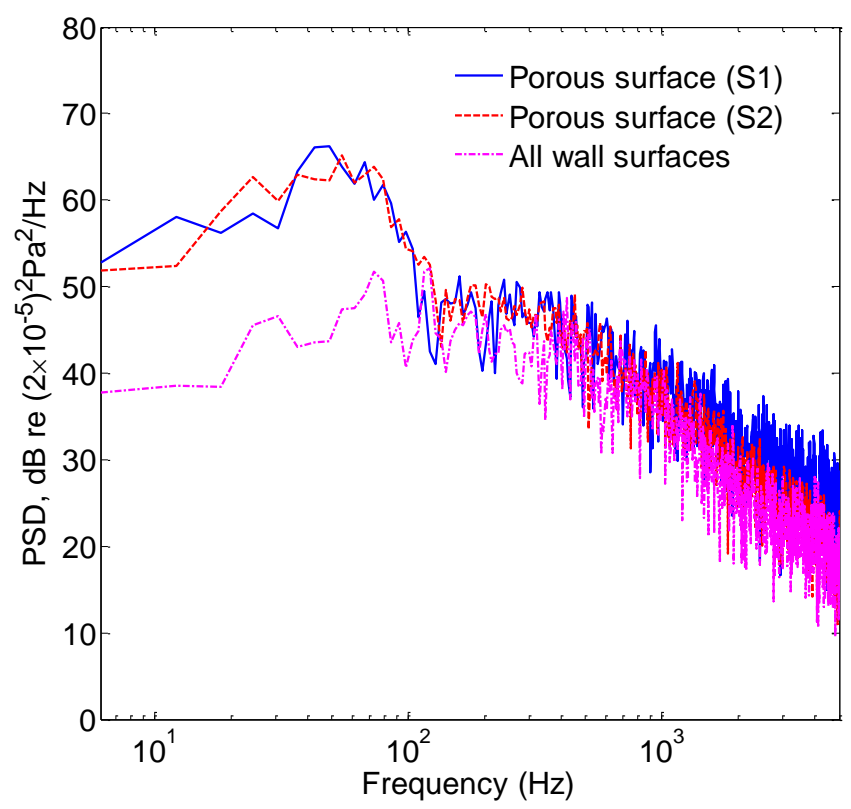

Figure 11. Comparisons of far-field noise spectra in the bogie cavity case without ground

Figure 12 shows the comparisons of the far-field noise predictions from the permeable surface (S1) for the cases with and without the ground, based on the corresponding flow simulation in each case. Figure 12(a) depicts the far-field noise spectra of these two cases. The receiver is again located $2.5 \mathrm{~m}$ away and $0.35 \mathrm{~m}$ above the bogie centre. It is found that the spectrum level is generally higher for the case with the ground underneath in most of the frequency range and the OASPL within the 
frequency range from $120 \mathrm{~Hz}$ to $2 \mathrm{kHz}$ is $74.2 \mathrm{~dB}, 7 \mathrm{~dB}$ higher than the case without the ground (OASPL of $67.2 \mathrm{~dB}$ ). Most of this difference is due to the reflection of sound in the ground plane. Figure 12(b) displays the noise directivity from the permeable surfaces for the two cases with and without the ground. The OASPL is calculated from the PSD over the frequency range from $120 \mathrm{~Hz}$ to $2 \mathrm{kHz}$ and the receivers are distributed uniformly around a semicircle with $2.5 \mathrm{~m}$ radius from the bogie centre in the bogie horizontal central plane. A similar lateral dipole pattern of noise radiation is predicted in each case, with noise levels around 6-8 dB higher for the case with the ground underneath compared with the case with no ground. This is caused by two effects. First, the ground reflection effect increases the sound radiation around $5.5 \mathrm{~dB}$ based on the calculations using the image method [22]. Second, there are more flow interactions for the case with the ground underneath, as discussed earlier, which increases the noise generation (about $1.5 \mathrm{~dB}$ contribution to the overall noise).

(a)

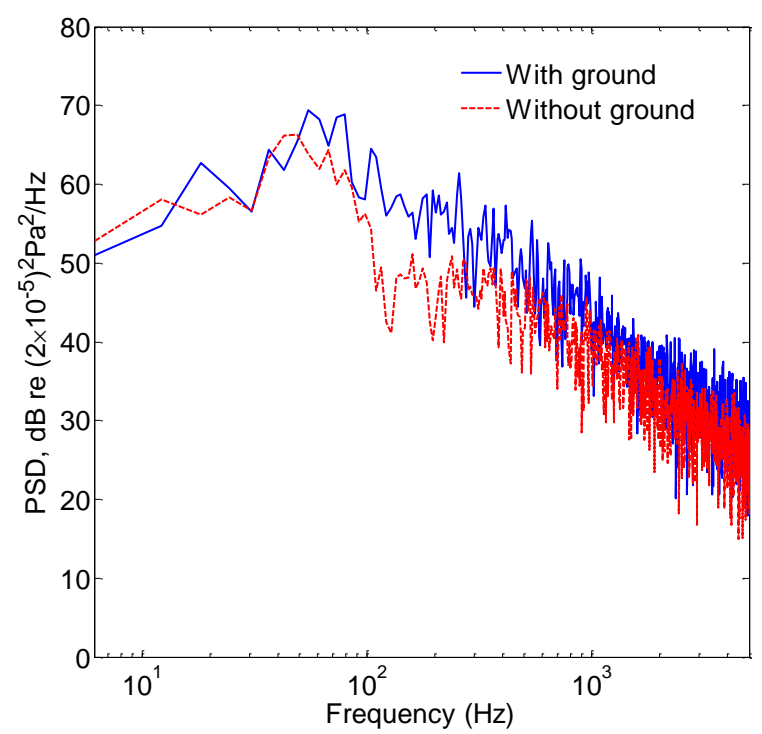

(b)

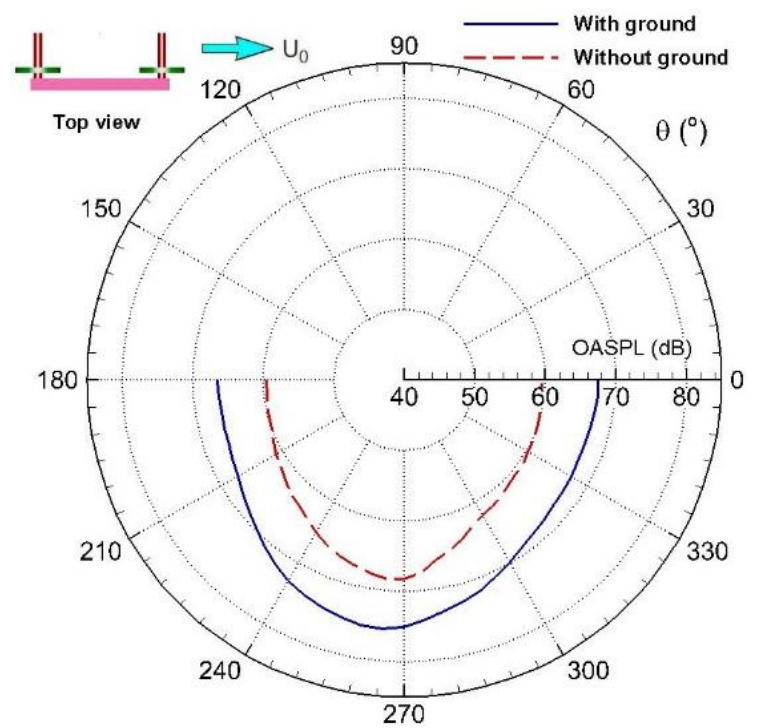

Figure 12. Comparisons of far-field noise prediction from the permeable surface: (a) noise spectra; (b) noise directivity.

\section{Conclusions}

The influence of the ground on the flow behaviour and the aerodynamic noise characteristics around a simplified bogie located inside the bogie cavity has been investigated using the DDES model and the FW-H acoustic analogy method. 
Firstly, the flow behaviour of an isolated wheelset with a stationary ground underneath has been studied numerically and experimentally at 1:5 scale for numerical verification. It is found that when the wheelset is close to the ground, an asymmetric flow pattern in the wheelset wake structure is formed. This is due to the shear flow separated from the lower part of the wheel which interferes with the boundary layer developed from the ground surface, and thus the upper separated flow is carried downwards since a pressure gradient is produced in the wheel wake region. The wheel wake shape predicted by the CFD simulation is verified by the PIV measurements.

Subsequently, the flow and flow-induced noise behaviour have been investigated of two cases of a bogie inside a cavity beneath a train car body with and without the ground. It is found that for both cases, a shear layer developed from the bogie cavity leading edge has a strong interaction with the flow separated from the bogie upstream components and the cavity wall. All vortices are mixed up and convected downstream and impinge on the downstream geometries and the cavity trailing edge as well as the rear wall regions. Thus, a highly irregular and unsteady flow is generated inside the bogie cavity due to the strong flow interactions and flow recirculations occurring there.

Additionally, the noise radiated to the trackside is predicted using a permeable data surface close to the bogie and parallel to the carbody side wall. Results show that a lateral dipole pattern of noise radiation is predicted. For the case with the ground underneath, the noise levels are around $7 \mathrm{~dB}$ higher as a consequence of more flow interactions compared with the case without the ground as well as the ground reflection effect.

This study at reduced scale has allowed insight to be obtained into the flow behaviour and aeroacoustic noise characteristics at full scale. However, it should be noted that for a full-scale case, the Reynolds number will be much higher and the more detailed geometry will lead to complex flow structures which will affect the noise generation and radiation. Nevertheless, the findings from the simplified bogie will be helpful to understand the aerodynamic noise generating mechanisms of the actual bogie in a high-speed train. 


\section{Acknowledgements}

The numerical simulations were performed on the Iridis4 cluster managed by the University of Southampton. The funding provided by the University of Southampton is gratefully appreciated. The data supporting this study are openly available from the University of Southampton repository at http://dx.doi.org/10.5258/SOTON/397952.

\section{References}

[1] Thompson DJ, Latorre Iglesias E, Liu XW, Zhu JY, Hu ZW. Recent developments in the prediction and control of aerodynamic noise from highspeed trains. International Journal of Rail Transportation, 2015, 3(3): 119-150.

[2] Thompson DJ. Railway noise and vibration: mechanisms, modelling and means of control. Elsevier, Oxford, UK, 2008.

[3] Mellet C, Létourneaux F, Poisson F, Talotte C. High speed train noise emission: Latest investigation of the aerodynamic/rolling noise contribution. Journal of Sound and Vibration, 2006, 293: 535-546.

[4] Nagakura K. Localization of aerodynamic noise sources of Shinkansen trains. Journal of Sound and Vibration, 2006, 293: 547-556.

[5] Herbst AH, Muld TW, Efraimsson G. Aerodynamic prediction tools for high-speed trains. International Journal of Rail Transportation, 2014, 2(1): 50-58.

[6] Yamazaki N, Kitagawa T, Nagakura K, Uda T, Wakabayashi Y. Prediction methods of aerodynamic bogie noise in wind tunnel test. Proc. 7th Forum Acusticum 2014, Krakow, Poland.

[7] Lauterbach A, Ehrenfried K, Kröber S, Ahlefeldt T, Loose S. Microphone array measurements on high-speed trains in wind tunnels. $3^{\text {rd }}$ Berlin Beamforming Conference, Berlin, Germany, 2010.

[8] Poisson F, Gautier PE, Létourneaux F. Noise sources for high-speed trains: A review of results in the TGV case. Noise and Vibration Mitigation for Rail Transportation Systems, Notes on Numerical Fluid Mechanics and Multidisciplinary Design, 2008, 99: 71-77.

[9] Spalart PR, Shur ML, Strelets MKh, Travin AK. Initial noise predictions for rudimentary landing gear. Journal of Sound and Vibration, 2011, 330, 4180-4195. 
[10] Zhu JY, Hu ZW, Thompson DJ. Flow simulation and aerodynamic noise prediction for a high-speed train wheelset. International Journal of Aeroacoustics, 2014, 13(7\&8): 533-552.

[11] Zhu JY, Hu ZW, Thompson DJ. Flow behaviour and aeroacoustic characteristics of a simplified high-speed train bogie. Proceedings of the Institution of Mechanical Engineers Part F: Journal of Rail and Rapid Transit, Published online September $30,2015$.

[12] Wang M, Freund JB, Lele SK. Computational prediction of flow-generated sound. Annual Review of Fluid Mechanics, 2006, 38: 483-512.

[13] Ask J, Davidson L. An acoustic analogy applied to the laminar upstream flow over an open 2D cavity. Comptes Rendus Mecanique, 2005, 333: 660-665.

[14] Spalart PR, Deck S, Shur ML, Squires KD, Strelets MKh, Travin AK. A new version of detached-eddy simulation, resistant to ambiguous grid densities. Theoretical and Computational Fluid Dynamics, 2006, 20: 181-195.

[15] Najafi-Yazdi A, Brès GA, Mongeau L. An acoustic analogy formulation for moving sources in uniformly moving media. Proceedings of the Royal Society of London, Series A, 2011, 467: 144-165.

[16] Orselli RM, Meneghini JR, Saltara F. Two and three-dimensional simulation of sound generated by flow around a circular cylinder. AIAA Paper 2009-3270, 2009.

[17] Schewe G. On the force fluctuations acting on a circular cylinder in crossflow from subcritical up to transcritical Reynolds numbers. Journal of Fluid Mechanics, 1983, 133: $265-285$.

[18] Zhu JY. Aerodynamic Noise of High-speed Train Bogies. PhD Thesis. University of Southampton, 2015.

[19] Ffowcs-Williams JE, Hawkings DL. Sound radiation from turbulence and surfaces in arbitrary motion. Philosophical Transactions of the Royal Society of London, 1969, 342: 264-321.

[20] Brentner KS, Farassat F. Modelling aerodynamically generated sound of helicopter rotors. Progress in Aerospace Sciences, 2003, 39: 83-120.

[21] Gloerfelt X, Bailly C, Juvé D. Direct computation of the noise radiated by a subsonic cavity flow and application of integral methods. Journal of Sound and Vibration, 2003, 266: 119-146.

[22] Dowling AP, Ffowcs-Williams JE. Sound and Sources of Sound. Horwood Publishing, Westergate, 1983. 